\title{
Genetic diversity and high proportion of intersubtype recombinants of HIV-I in urban Cameroon
}

\author{
Ana Machuca1, Shixing Tang*1, Jinjie $\mathrm{Hu}^{1}{ }^{1}$, Sherwin Lee ${ }^{1}$, Vutukuri Gupta ${ }^{1}$, \\ Leopold Zekeng ${ }^{2}$, Bih Awazi ${ }^{2}$, Chris Vockley ${ }^{1}$ and Indira Hewlett ${ }^{1}$
}

\author{
Address: ${ }^{1}$ Center for Biologics Evaluation and Research, Food and Drug Administration, Rockville, MD, USA and ${ }^{2}$ Mobile Laboratory, Sanitation \\ and Hygiene, Administration of Health, Cameroon \\ * Corresponding author
}

\author{
from 2006 International Meeting of The Institute of Human Virology \\ Baltimore, USA. 17-2I November, 2006 \\ Published: 21 December 2006 \\ Retrovirology 2006, 3(SuppI I):P6I doi:I0.II86/I742-4690-3-SI-P6I
}

(c) 2006 Machuca et al; licensee BioMed Central Ltd.

\section{Background}

A major characteristic of HIV-1 is its broad genetic diversity, which has significance for HIV transmission, treatment, diagnosis and prevention. We examined the genetic diversity of HIV in blood donors in Cameroon to understand the virus genotype distribution in this population.

\section{Methods}

A total of $88 \mathrm{HIV}-1$ positive samples, collected from blood donors in urban Cameroon in 2002, were genetically characterized in part of HIV-1 env (gp41) and gag (p17) regions through phylogenetic analysis.

\section{Results}

All the samples were infected with HIV-1 group M. No group $\mathrm{N}$ and $\mathrm{O}$ viruses were identified in the samples studied although they are endemic to Cameroon. HIV-1 infections were dominated by circulating recombinant form CRF02_AG (59\%) accompanied by additional group $M$ subtypes or CRFs: $A, D, F 2, G$, CRF01_AE, CRF13_Cpx and CRF22_01A1. A high percentage (26\%) of the samples had discordant subtype designations between gag and env, and appear to be unique intersubtype recombinant forms (URF) with the majority (91\%) involving recombination with either CRF01_AE or/and CRF02_AG. URFs containing CRF19 or CRF22 as well Fgag-Genv and Cgag-Aenv are reported. Some samples were not consistently detected by current HIV-1 testing assays.

\section{Conclusion}

Our study confirmed the existence of high number of cocirculating HIV-1 subtypes, the high intrasubtype diversity, and the high numbers of possible new recombinant viruses in urban Cameroon. These results highlight the epidemiologic importance of HIV-1 CRFs, especially CRF02_AG. 